\section{P54 Development of a Web-Based Nutrition Education Program for South Asians Using the DESIGN Procedure}

Zubaida Qamar, PhD, MS, zubqam@gmail.com, University of California, San Francisco (UCSF), 550 16th Street, 6th Floor, UCSF Box 1793, San Francisco, CA 94158

Objective: To describe an online, theory-based nutrition education program for South Asians in the U.S. and explain the rationale for design and implementation.

Theory, Prior Research, Rationale: South Asians (SA) have an increased risk of diabetes and cardiovascular diseases in comparison to most ethnic groups in the U.S. Scarcity of cost-effective, theory-based, nutrition education programs exist for SA emphasizing the need to develop and implement such programs.

Description: Program followed the DESIGN procedure and was guided by the Theory of Planned Behavior (TPB). The program constituted of 4 online lessons plans which were delivered on a weekly basis via email. Based on needs assessment, lessons focused on increasing fruit and vegetable (FV) intake (Lessons $1 \& 2$ ), physical activity and weight management (Lesson 3), label reading, portion control, healthy eating out strategies, and goal setting (Lesson 4). Web-based delivery was chosen as costeffective modality for this program. Multiple strategies were employed to recruit participants and participants were emailed validated and pre-tested pre/post surveys for completion. SA $(n=166$, mean age $=27.4 \pm 7.41,58.4 \%$ women) participants completed the pre-test survey out of which 66 signed up to participate. Email reminders were sent weekly to complete the lesson plans which contained information and educational exercises about the content.

Evaluation: The post-test survey assessed the evalution of the program, relationship of TPB constructs with intention and behavior, and nutrition strategies and health related behaviors. Out of these, the program significantly improved healthy eating strategies score, reduced fast food consumption, and increased self-efficacy towards FV intake. Participants (92\%) agreed or strongly agreed that program was overall helpful.

Conclusions and Implications: Program was well received and improved some healthy eating behaviors and mediators. Future work should focus on testing these program development and implementation strategies for cost-effective, large-scale nutrition education programs for the unique dietary considerations of ethnic groups in the U.S.

Funding: None.

\section{P55 Dietetic Internship as Learning} Community: A Project-Based Approach to Community Nutrition Education in Dietetics

Hannah Thornton, MS, LD, RDN, ht1074@txstate.edu, Texas State University, 601 University Drive, San Marcos, TX 78666; Lesli Biediger-Friedman, PhD, MPH, LD, RDN, Texas State University
Objective: To enhance understanding of and facility with community nutrition interventions among dietetic interns. Target Audience: Dietetic Interns.

Theory, Prior Research, Rationale: Learning communities foster collaborative inquiry about complex topics. Research has primarily focused on college-level learning communities, but recent evidence demonstrates the value of professional learning communities in fostering leadership and accountability. Project-based learning can improve students' ability to think critically and collaborate with their peers. Our research evaluates the effectiveness of learning community and project-based learning strategies at promoting professional confidence and leadership skills among dietetic interns.

Description of Course and Curriculum: Established within a university-based dietetic internship, the Food Security Learning Community (FSLC) includes graduatelevel dietetic interns, undergraduate dietetics students, and nutrition faculty. Faculty engage students in the development of policy, systems, and environmental approaches to food insecurity, guiding cohorts of students through a multi-year process that includes assessment of a problem, implementation of an intervention, and process and outcomes evaluation of the intervention. Students identify short-, intermediate-, and long-term goals, conceive of and assume autonomous leadership roles, create work plans, identify resources, and develop evaluation strategies. Learning goals established by the faculty are aligned with ACEND standards for dietetic internships. Interns receive alternate supervised practice hours for their work. The current FSLC focus is campus hunger. Year one included a mixedmethod assessment of student food security. Year two included implementation of a student-run, on-campus food pantry, laying the ground work for process and outcomes evaluation in year three.

Evaluation: Learning community and project-based learning strategies were evaluated through anonymous surveys, reflective writing, and semi-structured interviews completed by FSLC students. Results were analyzed using NVIVO and key themes were identified.

Conclusions and Implications: Preliminary results indicate overall satisfaction with the project, increased professional self-confidence, heightened awareness of leadership skills, and a sense of fulfillment. These strategies provide an educational framework that facilitates robust consideration of complex community nutrition problems by dietetic interns.

Funding: Texas State University.

\section{P56 Easy Choices: Making the Healthy Choice the Easy Choice}

Lisa Fuller, MS, LDN, RD, lfuller1@utk.edu, University of Tennessee Extension, 1801 Downtown West Boulevard, Knoxville, TN 37919; Clint Cummings, MS; Karen Franck, PhD; Christopher Sneed, PhD

Objective: To increase nutrition educators' ability to provide programming that promotes healthy behaviors for 